\title{
Paracervical abscesses as life-threatening complications of outpatient pain treatment
}

\author{
Report of three cases
}

\author{
Michael Schütze, M.D., ANd JÜRgen PIEK, M.D. \\ Department of Neurosurgery, University of Rostock, Germany
}

\begin{abstract}
The authors present three cases of iatrogenic paracervical abscesses caused by infiltration therapy of pain medications used in conservative treatment in patients with chronic cervicobrachialgia. All three patients had received this therapy several times before presenting with clinical signs of a local infection. The diagnosis was made based on results of the clinical examination, laboratory tests, and magnetic resonance imaging. The organism isolated was Staphylococcus aureus in all cases. After immediate surgery and long-term antibiotic therapy, all patients had a good outcome.

The authors conclude that infectious complications after invasive outpatient treatment often go undiagnosed, but as shown in these three cases can lead to acute and sometimes life-threatening complications. If the disease is diagnosed early, immediate surgical evacuation combined with antibiotic therapy results in recovery. For medicolegal reasons it should kept in mind that even a routine injection may lead to such complications.
\end{abstract}

\section{KEY WORDS - cervical abscess - complication • pain treatment - cervicobrachialgia}

\section{CASE REPORTS}

\section{Case 1}

History. This 60-year-old man was admitted to our department with progressive cervicobrachialgia in his left arm combined with local pain and a soft swelling in his left shoulder and upper chest. He had been treated as an outpatient for several weeks for a chronic cervicobrachialgia caused by degenerative cervical disc disease. During this treatment he had received repeated paracervical injections with unknown substances. A few weeks before this presentation he had become symptomatic and was treated twice with antibiotic medications for 2 weeks each time, and had also undergone a cervical incision for abscess evacuation. No further information could be obtained from the referring physician.

Examination and Operation. We obtained an MR image with Gd contrast material, which demonstrated a large left-sided paracervical liquid structure surrounded by contrast enhancement. On subsequent operation we found an encapsulated abscess containing creamy pus. The abscess was irrigated and drained.

Postoperative Course. Postoperatively the patient reported an immediate improvement of his pain and was discharged on a course of oral antibiotic therapy with clindamycin for 2 additional weeks. On follow-up review we found no recurrence; results of the neurological examination were normal.

Abbreviation used in this paper: $\mathrm{MR}=$ magnetic resonance.

\section{Case 2}

History. This 61-year-old man was admitted to our hospital with progressive cervicobrachialgia and a local swelling in the left cervical region. He had previously been treated for degenerative disc disease at the $\mathrm{C} 4 / 5$ level with repeated (three) paracervical injections. After the last infiltration he noticed progression of his pain and the beginning of cervical swelling with local redness and hyperthermia. The patient was admitted to our hospital based on findings on a cervical CT scan (Fig. 1).

Examination and Operation. Admission MR images ( $T_{1}$-weighted images with Gd contrast) demonstrated typical signs of paracervical abscess formation with a highintensity halo surrounding the center of a low-intensity area measuring $6 \times 3 \times 3 \mathrm{~cm}$. In the subsequent operation the abscess was removed by incision, irrigation, and drainage. Postoperative intravenous antibiotic therapy was initiated with clindamycin $(1800 \mathrm{mg} /$ day $)$ and continued for 1 week.

Postoperative Course. After immediate relief, the patient again experienced increasing pain in the treated region. Ultrasonography studies demonstrated only a small liquid structure, which was not reopened. Antibiotic therapy was continued, and after Day 11 the patient reported gradual improvement of his pain and was discharged. After 6 weeks he was free of pain and laboratory values (erythrocyte sedimentation rate, white blood cell count, C-reactive protein value) were nearly normal. The antibiotic therapy was continued until normalization of laboratory findings was achieved. 


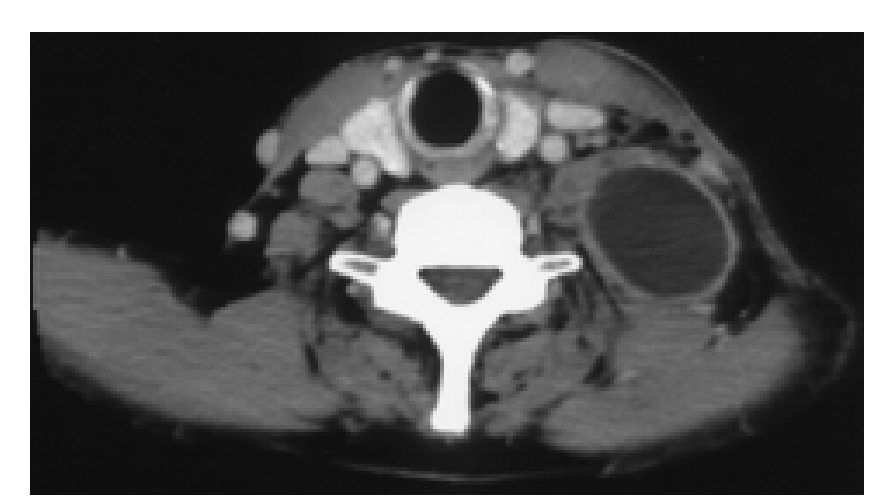

Fig. 1. Case 2. Preoperative computerized tomography scan obtained with contrast material, demonstrating typical signs of paracervical abscess formation with a high-intensity halo surrounding a center of low intensity measuring $6 \times 3 \times 3 \mathrm{~cm}$.

\section{Case 3}

History. After receiving several paracervical injections as an outpatient, this 58-year-old man was admitted to our unit for acute swelling in his right paracervical region and fever $\left(>39^{\circ} \mathrm{C}\right)$. The first injection had been performed 6 weeks before and the last had been given 8 days before admission. The indication for local pain treatment was chronic cervicobrachialgia after spondylosyndesis at C5-6 and C6-7 several years before and a persistent stenosis at the C4-5 level.

Examination. On clinical assessment we found an indurated swelling in the right paracervical region. Emergency MR imaging revealed a $2 \times 2.5 \times 3.5-\mathrm{cm}$ paracervical abscess at C3 -6 (Fig. 2).

Operation and Postoperative Course. Incision, irrigation, and drainage of the abscess was performed immediately. Postoperatively the patient reported rapid improvement of his pain. He was also started on a regimen of intravenous clindamycin $(1800 \mathrm{mg} /$ day $)$ and was discharged on a course of oral antibiotic therapy 1 week later. Six weeks later the patient was readmitted with the same symptoms. For unknown reasons his referring physician had discontinued the antibiotic therapy 2 weeks earlier. The patient underwent repeated surgery in the same manner. After 10 days he was discharged and was symptom

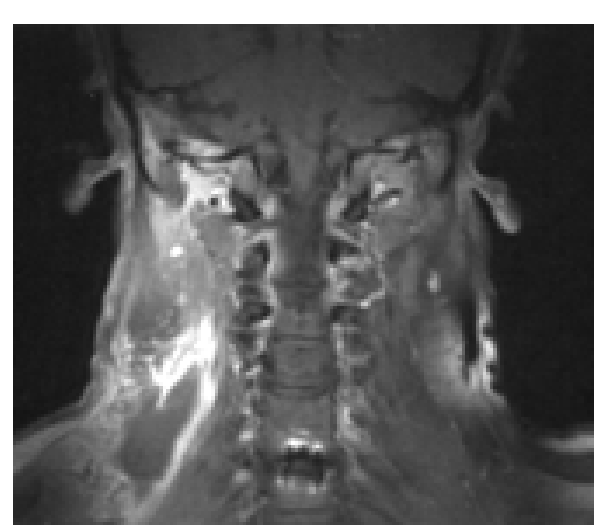

Fig. 2. Case 3. Preoperative MR image ( $\mathrm{T}_{1}$-weighted with $\left.\mathrm{Gd}\right)$ demonstrating a paracervical abscess measuring $2 \times 2.5 \times 3.5 \mathrm{~cm}$ at C3-6. free. Clindamycin therapy was continued until normalization of laboratory parameters occurred.

We were unable to identify the substances that had been injected for pain treatment in any of the three patients. In Germany substances like a local anesthetic agent combined with a corticoid drug and hyaluronic acid are often injected in such cases. Tissue cultures were obtained in all three patients, and all of them showed $S$. aureus as the cause of infection.

\section{DISCUSSION}

Repeated paracervical injection for treatment of chronic cervicobrachialgia is a well-known and frequently performed outpatient procedure. To our knowledge, no studies of iatrogenic infections occurring as a complication of such procedures have been published. Each injection, however, carries a certain risk of infection. ${ }^{3,4}$ For example, infectious complications with epidural catheters are well known; the infection rate is approximately $1: 100,000$ to $1: 400,000$ cases in patients receiving spinal epidural anesthesia. ${ }^{2}$ Therefore, patients who undergo this procedure must be informed of such risks and informed consent is mandatory.

In previous reports infections related to epidural catheters have often been associated with disease elsewhere in the body, particularly skin and soft tissue infections. ${ }^{1}$ In our series there was no evidence of infection elsewhere at the time of the initial treatment. There was also no sign of underlying immunocompromise, which might have predisposed the patients to abscess formation.

As a result we conclude that infectious complications after invasive outpatient treatment may go undiagnosed because paracervical injections are regarded as harmless procedures. Nevertheless, as shown in our three cases, infections can occur and can lead to acute and sometimes life-threatening complications. If diagnosed immediately, surgical evacuation combined with antibiotic therapy results in recovery. Antibiotic therapy should be aimed at the most likely causative organism (that is, S. aureus). Short-term antibiotic regimens may lead to recurrence (as in Case 3). Therefore we advocate oral antibiotic therapy until laboratory values (erythrocyte sedimentation rate, Creactive protein level, white blood cell count) return to normal. For medicolegal reasons it should be kept in mind that even a harmless injection may result in severe complications.

\section{References}

1. Danner RL, Hartman BJ: Update on spinal epidural abscess: 35 cases and review of the literature. Rev Infect Dis 9:265-274, 1987

2. Dunn LT, Javed A, Findlay G, et al: Iatrogenic spinal infection following epidural anaesthesia: case report. Eur Spine J 5: 418-420, 1996

3. Fradis M, Goldsher M, David JB, et al: Life-threatening deep cervical abscess after infiltration of the tonsillar bed for tonsillectomy. Ear Nose Throat J 77:418-421, 1998

4. Huang RC, Shapiro GS, Lim M, et al: Cervical epidural abscess after epidural steroid injection. Spine 29:E7-E9, 2004

Manuscript received October 25, 2004.

Accepted in final form November 4, 2004.

Address reprint requests to: Michael Schütze, M.D., Department of Neurosurgery, University of Rostock, Schillingallee 35, 18057 Rostock, Germany. email: michael.schuetze@med.uni-rostock.de. 